\title{
Bilateral primary malignant lymphoma of the breast
}

\author{
B. Shpitz, M. Witz, Z. Kaufman, B. Griffel, Y. Manor and A. Dinbar \\ Departments of Surgery 'B', Pathology, and Medicine 'D', Meir General Hospital, Kfar-Saba, and the Tel Aviv \\ University Sackler School of Medicine, Tel Aviv, Israel.
}

\begin{abstract}
Summary: A rare case of bilateral primary malignant lymphoma of breast in a 76 year old woman is presented. The lesion was examined by electron microscopy and immunochemistry. The diagnosis of primary malignant lymphoma remains a diagnosis by exclusion and requires extensive work-up to exclude widespread malignant process. The behaviour of this malignancy tends to be an aggressive one and the prognosis is generally poor.
\end{abstract}

\section{Introduction}

Primary malignant lymphoma of breast (PMLB) is a rare disease. According to data taken from the End Results Group of Cancer Registries (Freeman et al., 1972), 33 cases of breast lymphoma were registered among 1,467 extranodular lymphomas. Bushkin et al. (1973) reported only 113 cases from the world literature up to the year 1972 . Only about $6 \%$ of the patients with PMLB had bilateral involvement (Freedman et al., 1971). A rare case of bilateral PMLB is presented here.

\section{Case report}

A 76 year old woman was referred to our department for bilateral breast biopsy of symmetric non-tender lesions of a few months duration in the upper-external quadrants of both breasts. Seven years previously she underwent right hemicolectomy for adenocarcinoma of the caecum.

On examination, bilateral, symmetrical, mobile, round, non-tender, irregular lesions, $5 \times 5 \mathrm{~cm}$ and $3 \times 3 \mathrm{~cm}$ in diameter, were found in the upper-external quadrants of the breasts. There were no palpable axillary or supraclavicular lymph nodes. The remaining physical examination was normal. Laboratory examinations were within normal limits.

The patient underwent bilateral lumpectomy. The lumps received from both breasts for histopathological examination were $7 \times 5 \times 4 \mathrm{~cm}$ and $5 \times 4 \times 2 \mathrm{~cm}$. They were composed of white, homogeneous, rubbery tissue, with small areas of fat tissue in the lumps and in the periphery.

Correspondence: A. Dinbar, M.D., Department of Surgery 'B', Meir General Hospital, 44281 Kfar Saba, Israel.

Accepted: 31 January 1985
Microscopically, a severe diffuse infiltration of dark cells was seen in both lumps. The infiltrate varied in its density; around the ducts it was more dense and in between these areas less so (Figures 1 and 2). The cells had small, round nuclei, mainly very dark, without nucleoli or signs of mitosis. Some of the cells were larger and had a clearer, more oval nucleus.

The slides were all negative for immunoglobulins IgM, IgG, IgA, IgE and lysosome by the peroxidaseanti-peroxidase method (Dakopatt, Denmark). No antibodies for $\mathrm{T}$ cells were available for staining with the same method.

On electron microscopic examination, the tumour cells had very irregular nuclei, scanty cytoplasm with a normal amount of organelles as mitochondriae, vesicles and ribosomes. No desmosomes were recognized.

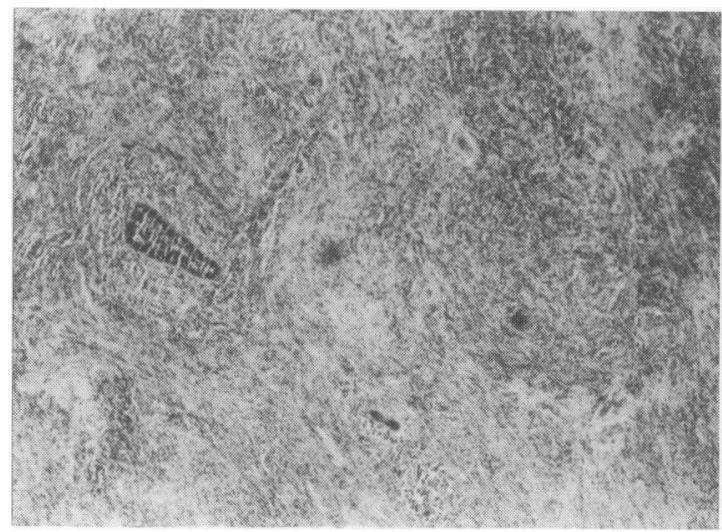

Figure 1 Low-power picture of the infiltrate. The dark areas are mammary ducts. (H \& $\mathrm{E} \times 40)$.

(C) The Fellowship of Postgraduate Medicine, 1985 


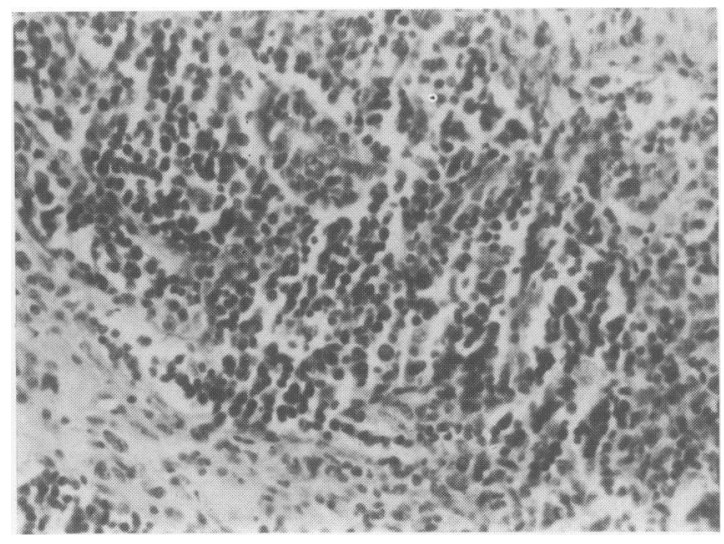

Figure 2 Dense lymphomatous infiltrate around one ductule. (H \& $\mathrm{E} \times 250)$.

Around the ducts the cells were in direct contact with the thickened basal membrane.

Judging by the light microscopic picture and by electron microscopy findings, the diagnosis of malignant lymphoma, diffuse large cell type, was made (using 'The Working Formulation for Clinical Usage' of the Non-Hodgkin's Lymphoma Pathologic Classification Project, 1982. Because of the negative immunochemistry for immunoglobulins, the possibility of $T$ cell or null cell lymphoma arose, but could not be proved.

The post-operative work-up, including bone marrow biopsy and computed tomographic (CT) scan, did not reveal any residual malignant process.

Post-operatively, the patient received adjuvant chemotherapy by CHOP (cyclophosphamide, Adriamycin, vincristine, prednisone) every 3-4 weeks for 9 months, followed by COP (cyclophosphamide, vincristine, prednisone).

One year after surgery the patient underwent reevaluation, including CT scan, which showed no pathological findings. She still receives COP chemotherapy and is in complete remission 20 months after initial diagnosis.

\section{Discussion}

PMLB is a rare disease, comprising only 0.12 to $0.53 \%$ of all breast malignancies, and 10 to $20.6 \%$ of sarcomas of the breast (Mambo et al., 1977; Wiseman \& Liano, 1972). Lawler \& Ritchie (1966) reviewed the world literature up to 1966 and found 61 cases of this malignancy, including one of their own, the only case of PMLB out of 3,608 neoplasms of the breast reported by Vanderbilt University Hospital in a period of $36 \mathrm{y}$. Bushkin et al. (1973) found 113 cases of PMLB in the world literature up to 1972. Freeman et al. (1972) reported $0.07 \%$ of PMLB among 1,467 extranodular lymphomas. The reported incidence of bilaterality is about 6\% (Freedman et al., 1965).

The chief complaint reported by most authors was a rapidly growing mass (Bushkin et al., 1973; Mambo et al., 1977; Yoshida, 1970). Clinically it is impossible to differentiate between this malignancy and carcinoma. Because of the lack of specific clinical signs and the rarity of the disease, the diagnosis was not made preoperatively in most cases (Mambo et al., 1977; De Cosse et al., 1962). However, a rapidly enlarging mass and multiple synchronous lesions can be suspected of being a malignant lymphoma of the breast (Bushkin et al., 1973; Lawler \& Ritchie, 1967; Yoshida, 1970).

From a review of the English literature we found that Wiseman \& Liao (1972) offered the first strict and adequate criteria for diagnosis of PMLB, excluding those cases in which the breast was the initial presentation of an already wide-spread malignant process.

Our patient, who underwent extensive work-up, including bone marrow biopsy and CT scan, represented a case of PMLB, fulfilling the following criteria (in accord with those outlined by Wiseman \& Liao): (a) lack of previous extramammary lymphoma; (b) lymphoma tissue found within breast tissue, and (c) documentation of breasts as a primary clinical site, with adequate follow-up.

Different methods of treatment have been employed over the last $30 \mathrm{y}$. Most authors held that the treatment of choice for PMLB should be either simple or radical mastectomy with regional irradiation (Freedman et al., 1971; Wiseman \& Liao, 1972; Lawler \& Ritchie, 1967; De Cosse et al., 1962). In contrast, most of the patients in the series of Mambo et al. (1977) had excisional biopsy or wedge resection and postoperative chemotherapy, with or without regional irradiation to the tumour area, axillary and supraclavicular lymph node groups. The type of adjuvant treatment used was either single or combined chemotherapy by cyclophosphamide, Adriamycin, vincristine, prednisone and bleomycin. According to Yoshida's review (1970), adjuvant chemotherapy after radical mastectomy was also used in Japan in 6 of 13 reported patients with PMLB.

Both radical and conservative surgery have been reported to have long term survivors (Mambo et al., 1977; Lawler \& Ritchie, 1967; De Cosse et al., 1962). In view of the fact that this disease tends to progress to a systemic malignant process in a significant number of patients, it seems that a conservative surgical approach (breast biopsy or lumpectomy) is justified on the one hand, but a more aggressive adjuvant chemotherapy by means of multi-agent chemotherapy with or without irradiation is indicated on the other 
hand. Following this approach, our patient was treated by bilateral lumpectomy continued by adjuvant chemotherapy.

The prognosis of patients with PMLB is relatively

\section{References}

BUSHKIN, F.L., DEMIAN, S.D.E. \& PIERSON, K.K. (1973). Primary reticulum cell sarcoma of the breast. American Journal of Surgery, 126, 63.

DeCOSSE, J.J., BERG, J.W., FRACCHIA, A.A. \& FARROW, J.H. (1962). Primary lymphosarcoma of the breast. A review of 14 cases. Cancer, 15, 1264.

FREEDMAN, S.I., KAGAN, A.R. \& FRIEDMAN, N.B. (1971). Bilaterality in primary lymphosarcoma of the breast. American Journal of Clinical Pathology, 55, 82.

FREEMAN, C., BERG, J.W. \& CUTLER, S.J. (1972). Occurrence and prognosis of extranodal lymphomas. Cancer, 29, 252.

LAWLER, M.R. \& RITCHIE, R.E. (1967). Reticulum cell sarcoma of the breast. Cancer, 20, 1438. unfavourable. The survival rates, however, differ in reported series, ranging from $50 \%$ of 1 year survival to $54 \%$ of 10 year survival (Lawler \& Ritchie, 1967; De Cosse et al., 1962).

MAMBO, N.C., BURKE, J.S. \& BUTLER, J.J. (1977). Primary malignant lymphomas of the breast. Cancer, 39, 2033.

THE NON-HODGKIN'S LYMPHOMA PATHOLOGIC CLASSIFICATION PROJECT (1982). National Cancer Institute sponsored study of classifications of non-Hodgkin's lymphomas. Summary and description of a working formulation for clinical usage. Cancer, 49, 2112.

WISEMAN, C. \& LIAO, K.T. (1972). Primary lymphoma of the breast. Cancer, 29, 1705.

YOSHIDA, Y. (1970). Reticulum cell sarcoma of the breast. Case report and a review of the Japanese literature. Cancer, 26, 94. 